Results A total of 26 professionals participated in the interviews. The main facilitator for implementation of the CDSS was considered to be easy access to well-structured patient data, and the resulting reduction of MDTM preparation time and of duration of MDTMs. Less impact of the CDSS was expected on the quality of lung cancer services generated by MDTM decision-making. Main barriers for adoption included incomplete or non-trustworthy output generated by the system and insufficient adaptability of the system to local and contextual needs. Actionable findings for an implementation strategy were a usability test involving key users and a validation study in the organization's real-life setting prior to roll out.

Conclusion Using this CDSS in lung cancer MDTMs was expected to increase efficiency of workflows. Successful implementation is dependent on the reliability and adaptability of the CDSS and involvement of key users in the implementation process.

\section{COLLABORATIVE QUALITATIVE RESEARCH ON SUICIDE AND SELF-HARM IN SOUTH ASIA: A REFLECTION ON CHALLENGES AND SOLUTIONS}

${ }^{1}$ A Krayer, ${ }^{2} \mathrm{~S}$ Das. ${ }^{1}$ School of Health Sciences, Bangor University, UK; ${ }^{2}$ SASHI project, Mysore, India

\subsection{6/bmjopen-2021-QHRN.18}

Trust is essential to planning and delivering impactful international research that is culturally appropriate and has the potential to change practice and policy on local levels. However, details on how this is can be achieved, and a discussion of challenges encountered are often lacking. A better understanding of building and maintaining of trust in North-South research partnerships is essential, especially when tackling complex and sensitive issues such as self harm and suicide. Suicide is amongst the leading causes of death in South Asia.

This talk will reflect on experiences in the South Asia SelfHarm Initiative (SASHI), a global-challenges funded research project, led by co-investigators from the Global North and South. The research collects empirical evidence to inform the understanding of the nature of self-harm in the context of profound social, political and economic challenges in the global South as well as builds research capacity. We draw on Ben-Ari and Enosh's work (2010), which focuses on identifying incongruities that challenge our knowledge (discovery) and examine them in-depth as a source of new knowledge (construction) to come to a new understanding. The definition of trust is debated, and our starting point is Luhmann's (1999) approach that trust is expressed through social action in contexts we cannot fully know.

We argue that trust is a building block for fair and equitable international research partnerships and is continually developed and negotiated in relationships and activities. Power inequalities and contextual factors need to be acknowledged. Working on building and maintaining trust is emotionally and cognitively challenging. Our experiences suggest that building and maintaining trust relies on recognising similarities, which can foster respect and equality of status. Acknowledging and exploring differences can provide opportunities for reflection and joint learning. These issues are important to consider as they ultimately shape knowledge production and translation.
THE DYNAMICS OF TRUST AND STRUCTURAL COERCION WITHIN A MENINGITIS TRIAL IN SUB-SAHARAN AFRICA

${ }^{1} \mathrm{D}$ Lawrence, ${ }^{2} \mathrm{~A}$ Ssali, ${ }^{3} \mathrm{~K}$ Tsholo, ${ }^{1} \mathrm{~J}$ Jarvis, ${ }^{1} \mathrm{~J}$ Seeley. 'London School of Hygiene and Tropical Medicine, UK; ${ }^{2}$ MRC/UVRI and LSHTM Uganda Research Institute, Uganda; ${ }^{3}$ Botswana Harvard AIDS Institute Partnership, Botswana

\subsection{6/bmjopen-2021-QHRN.19}

Background Clinical trials in sub-Saharan Africa typically offer better medical care than is routinely available. This can lead to structural coercion where an individual may consent because of a lack of alternative options and potentially despite being uncertain about the research. An inherent component of this decision making process is an assessment of trust. Trust in the treatment options, the research team, and the process as a whole. This may be polarised in the context of life-threatening illnesses where recruitment (or not) could determine survival.

Aim We sought to understand the dynamics of trust and structural coercion in a multi-site clinical trial for HIV-associated cryptococcal meningitis.

Methods We embedded an ethnographic study within a clinical trial for HIV-associated cryptococcal meningitis. We conducted in-depth interviews with trial participants and their next-of-kin in Uganda and Botswana. We combined these with direct observations and in-depth interviews with researchers working at the African sites and European partner institutions. Interviews were transcribed, translated, and subject to narrative analysis.

Results To date we have recruited 14 trial participants, five next-of-kin and ten researchers. Recruitment is on-going until March 2021. Participants and their relatives often felt they had no choice but to enrol in the clinical trial which was their best chance of survival. Despite the perceived benefits of participation, recruitment came at a cost to participants who agreed to invasive medical procedures such as lumbar punctures despite pre-existing beliefs they could cause death. The severity of the illness contributed to poor comprehension of what the trial entailed and the decision to participate was heavily based on trust in the research team.

Conclusions Structural coercion is a significant factor impacting recruitment into clinical trials in resource-limited settings. In the context of life-threatening illness, trust superseded the need for an in-depth understanding of the research process.

\section{TRUST AND RELIANCE WITHIN SPECIALIST CLINICAL SERVICES: COUNTER-PRODUCTIVE OR HELPFUL FOR SELF-MANAGEMENT OF PEOPLE WITH NEUROMUSCULAR CONDITIONS?}

${ }^{1}$ LE Lee, ${ }^{2}$ ST Kulnik, ${ }^{2}$ A Boaz, ${ }^{3} \mathrm{G}$ Ramdharry. 'Department of Neuromuscular Diseases, UCL Queen Square Institute of Neurology, UK; ${ }^{2}$ Faculty of Health Social Care and Education, Kingston University and St George's University of London, UK; ${ }^{3}$ Department of Neuromuscular Diseases, UCL Queen Square Institute of Neurology, UK

\subsection{6/bmjopen-2021-QHRN.20}

Current approaches to self-management de-emphasise dependency on healthcare services and focus on building confidence and capability. Our qualitative study explores self-management perspectives from individuals with neuromuscular conditions who attend regional specialist clinics, to inform implementation of a self-management intervention. 
Data indicate high levels of trust and reliance upon clinicians' expert knowledge and accessibility of the service, flagging up potential tensions with self-management principles of valuing patient-held expertise, and fostering individuals' knowledge and skills to self-manage. Somewhat paradoxically, participant's geographical spread, combined with lack of specialists in local services, acts as both a driver to self-management, and towards reliance on specialist centres. Can increased reliance on specialist services be reconciled with self-management principles?

Twelve individuals were recruited from specialist clinics and participated in semi-structured interviews. Data were analysed against self-management principles informed by Social Cognitive Theory, and a tripartite theoretical conceptualisation of trust involving reliability, general, and specific trust. Data were then examined for incongruity and potential impact on implementation.

Service-users' trust in specialist services arises, firstly, from clinicians' understanding and knowledge of neuromuscular conditions, resulting in information perceived as good-quality and reliable; and secondly, from continuity and accessibility of care. This may naturally play towards health service-centred narratives, which are incompatible with self-management implementation. However, the data support alternative interpretations to align trust and reliance on specialist services with self-management. Opportunities for vicarious learning are sparse in rare conditions, but clinicians can promote self-efficacy through vicarious dialogue, and sharing experiences of other service-users. Trust acts as an enabler to goal-setting and positive risk-taking. Fundamentally, trust in the service may constitute a necessary pre-requisite without which a self-management dialogue is likely to be rejected.

Our preliminary findings highlight the uniqueness of patient-clinician relationships in this setting, providing insights into how the intervention can be implemented to this clinical context and population.

\section{THERAPEUTIC SETTINGS AND BEYOND: A TASK ANALYSIS OF RE-ESTABLISHING EPISTEMIC TRUST IN PSYCHOTHERAPY}

${ }^{1} \mathrm{E}$ Li, ${ }^{1} \mathrm{~N}$ Midgley, ${ }^{1} \mathrm{P}$ Luyten, ${ }^{2} \mathrm{C}$ Campbell. ' UCL; Anna Freud National Centre for Children and Families, UK; ${ }^{2}$ University College London, UK

\subsection{6/bmjopen-2021-QHRN.21}

Background The concept of epistemic trust captures one's willingness to receive new information as trustworthy and relevant, underpinning one's learning capacity to internalize new knowledge and generalize them in a wider context. The lack of epistemic trust may link to the emergence and persistence of general psychopathology. As a recently introduced concept in developmental and clinical psychology, epistemic trust requires empirical work never previously done.

Aims The aim is to explore how epistemic mistrust (e.g., hyper vigilance, petrification) come about in one's development leading to mental disturbance, how epistemic trust can be (re-)established in psychotherapy, and moreover, how therapists act can appropriately respond to epistemic mistrust and help establish epistemic trust.

Methods Task analysis, an inductive approach where patterns of change can be identified within a psychotherapy context, will be conducted in randomly selected audiorecordings of psychotherapy sessions from the Improving Mood with Psychoanalytic and Cognitive Behaviour Therapy (IMPACT) study, a randomized controlled trial comparing three interventions in the treatment of depression in adolescents.

Expected results A model of (re-)establishing epistemic trust in psychotherapy for depressed adolescents will be presented by engaging cases with both good and poor outcomes, with an awareness of the potentially different productive processes used across three treatment arms. The results answer whether levels of epistemic trust moderate one's psychotherapy outcomes and propensity to thrive in socially adverse circumstance-allow the extent to which epistemic trust underpins resilience to be further understood. Moving beyond specific mental disorders and psychotherapy orientations, we will argue how a lack of epistemic trust is common in general psychopathology and its restoration is necessary for all kinds of treatments based on our findings to help guide the professionals in practice.

The current stage of the work: Writing a study protocol.

\section{ENGAGING COMMUNITY: WAYS TO IMPROVE TRUST AND OUTCOMES THROUGH COMMUNITY-LED METHODS AND ANALYSIS}

K Luxion, M Svensson, D Frost. University College London, UK

\subsection{6/bmjopen-2021-QHRN.22}

Within the context of low-resource projects, both limited on time and resources, it can be difficult to incorporate equitable research methods, such as community-based participatory research. For LBGTQ+ research, there are similar barriers, with the need for health research projects that support community-led projects and centre LGBTQ+ voices through their methods. This presentation frames research methods as a social justice issue, addressing the value of community members as academic researchers; it seeks to highlight additional ways in which community involvement increases trust in both research processes and outcomes. The included examples are sub-projects and methods that are part of two distinct LGBTQ+/community-led PhD projects using either a multi-study or mixed methods approach; as such the discussion is focused on their use of qualitative methods. Coming from a social psychology perspective, themes are related to plurisexual emerging adults' parenthood desires and plans and a biomedical study on LGBTQ + pregnancy and parenthood, with recommendations that are transferable to other health topics. Focusing on the primary example, the presentation discusses the benefits of engaging LGBTQ+ community members assist on data processing and meaning-making while building qualitative research skills. A case study based on this postgraduateundergraduate mentoring scheme, proposes a purposeful approach to encouraging community-led research as part of capacity building efforts for undergraduates, within and beyond academia. Information to be covered, in addition to the case study, includes how participant trust was fostered through protocols, methods, and the analysis process - with further examples pending. 